\title{
A Sign of Trust? The value of source attribution in accolade claims
}

\section{Mathew S. Isaac}

Professor

Seattle University

Albers School of Business and Economics

isaacm@seattleu.edu

\section{INTRODUCTION}

When designing effective on-premise or outdoor signs, advertising agencies routinely stress the importance of simplicity. Clear Channel Outdoor, the oldest outdoor advertising company in the United States, notes on its website: "Simplicity is the fundamental guideline for creating good out-of-home design. The most effective out-of-home designs capture the essence of a message with lucid expression" (Clear Channel 2019). This fundamental design principle applies not only to outdoor signs, but also to on-premise signs. In its best practice standards, the United States Sign Council Foundation advocates short messages and simple typography for on-premise signs as those require less time to read and mentally process (Garvey et al. 2018). Academic research has corroborated the view that greater visibility (achieved through conspicuousness and legibility), in concert with lower informational density and complexity, usually enhances the effectiveness of a sign as a communication vehicle (see Bullough 2017; Stempler and Polger 2013; Van Loock, Vermeir, and Geuens 2010).

Signs, however, are often meant not only to educate and inform, but also to change attitudes and persuade potential customers. In such situations, richer and more detailed content-with greater informational density and complexity-may be more effective because it offers greater specificity and enhanced credibility (MacInnis et al. 1991; Morrison and Dainoff 1972; Phillips 1997). I contend that there is an inherent tension between simplicity and credibility in certain types of persuasive signage. The present research focuses specifically on signs that contain accolade claims, those which tout endorsements, awards, or honors that an organization has receivedpresumably from a third-party entity or accrediting body. When sharing news of its accolade with current or prospective customers, an organization must make decisions related to source attribution - that is, how much detail

\section{Abstract /}

Organizations that have received an accolade or honor often share this information with current or prospective customers, either in a digital (i.e., on their website, social media, etc.) or physical (i.e., on-premise signs, outdoor signs, etc.) format. When publicizing their achievement, marketers must make decisions related to source attribution-that is, how much detail to provide and how prominently (if at all) to mention the thirdparty entity that bestowed the accolade upon them. This is an important question, particularly with respect to physical signs where informational complexity is often detrimental and visual clarity is paramount. In this research, I examine whether source attribution in signage materially affects consumer evaluations and behavioral intentions. Across three studies, I find converging evidence that source attribution in accolade claims does in fact bolster evaluations and behavioral intentions, even in the context of physical signage when consumers are likely to be engaged in heuristic processing. Furthermore, I provide evidence that these more positive judgments arise because attribution increases perceived credibility of the organization receiving the accolade.

\section{Keywords /}

awards; credibility; source attribution; heuristic processing; advertising claims 
to provide and how prominently (if at all) to mention the third-party entity that bestowed the accolade. For example, after being named by Food and Wine magazine as one of the best new restaurants of 2020, Nixta Taqueria might consider posting a high source attribution sign with detailed information about the accolade and the source or a low source attribution sign with limited information (Shah 2020). In the context of signage, I examine whether consumer attitudes and behaviors are materially influenced by the level of source attribution in an accolade claim.

\section{ACCOLADE CLAIMS}

Organizations whose products or services have received endorsements, awards, or honors from a third-party entity often share this information with current or prospective customers (see Isaac, Brough, and Grayson 2016). Although such accolades may be communicated digitally, they are routinely publicized via physical signs. Figures $1 \mathrm{~A}$ and $1 \mathrm{~B}$ respectively provide examples of outdoor signs and on-premise signs in which organizations have communicated accolades that they received.

The proliferation of accolade claims in signage suggests a belief among practitioners that such claims will have positive downstream consequences on consumer attitudes and purchasing behavior. This assumption is supported by prior research showing that consumers' evaluations tend to increase when they learn that an organization has received an honor or award (Balasubramanian, Mathur, and Thakur 2005; Dean and Biswas 2001).
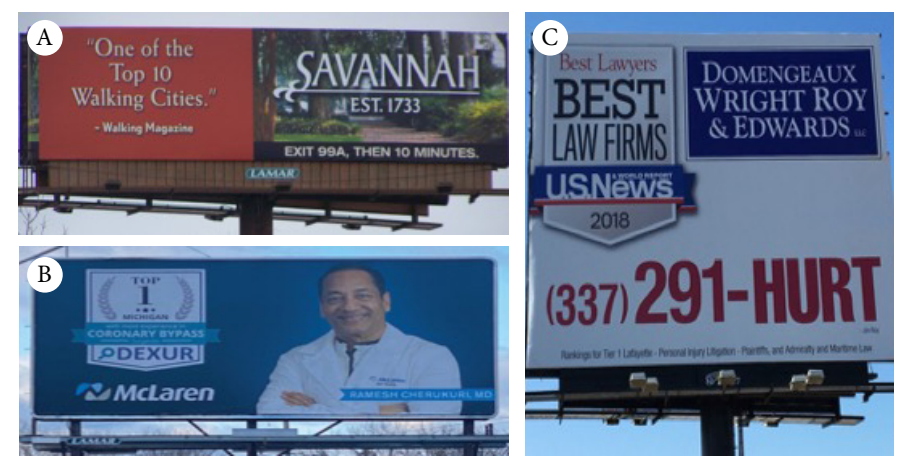

Figure 1A / Example (A): Top Walking Cities sign, Savannah, Georgia; (B): Top Doctor sign, Bay City, Michigan ; (C): Best Law Firm sign, Lafayette, Louisiana
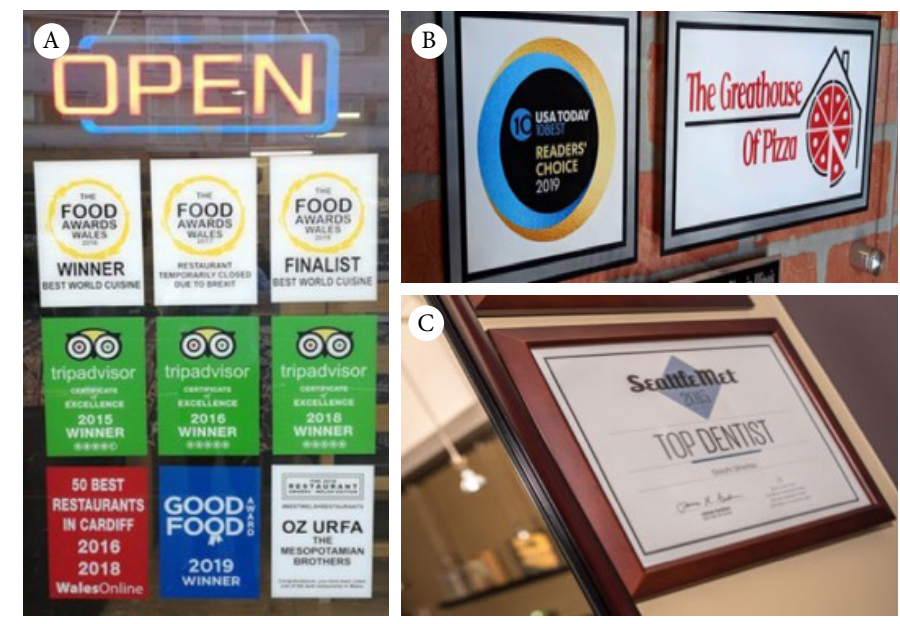

Figure 1B / Example (A): OZ Urfa Restaurant sign, Cardiff, Wales; (B): The Greathouse of Pizza sign, Casey, Illinois; (C): Goichi Shiotsu Dentistry sign, Mercer Island, Washington

Importantly, accolade claims vary considerably with respect to the level of detail provided about the accolade and even the third-party entity that conferred the accolade. The provision of fewer details is especially likely for signage, where physical space is at a premium and organizations may feel compelled to adhere to the principle of simplicity. Figure 2 provides examples of signage where the identity of the third-party entity who conferred the accolade to the organization is absent. Such claims are considered low in source attribution.
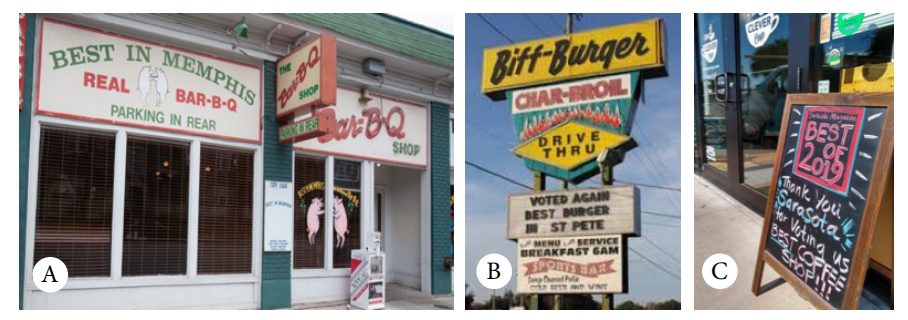

Figure 2 / Example (A): Bar-B-Q Shop Restaurant sign, Memphis, Tennessee; (B): Biff-Burger Restaurant sign, St. Petersburg, Florida; (C): The Clever Cup Coffee Shop sign, Sarasota, Florida

\section{THE CASE FOR LOW SOURCE ATTRIBUTION}

Within the context of physical signs, are consumers sensitive to source attribution? The answer to this question is not straightforward because of the inherent tradeoff between simplicity and specificity in marketing communications-a tradeoff that may 
be particularly pronounced in signage. According to the outdoor advertising company Capitol Outdoor, the average person views a billboard for about four seconds (Capitol 2020). As such, consumers may not have sufficient time to encode detailed source attribution information.

Furthermore, there is limited real estate on a physical sign and marketers must therefore be judicious in determining the content that merits inclusion. Adding source attribution information may make a sign appear more cluttered, which can interfere with conspicuousness and legibility (Bullough 2019). Prior work, largely in advertising, has repeatedly documented the benefits of simplicity in marketing communications (Anderson and Jolson 1980; Chamblee et al. 1993; Lowrey 1998; Morrison and Dainoff 1972; Rossiter and Percy 1983; Shuptrine and McVicker 1981). Research specifically on signage has also shown that cluttered and illegible signs tend to be ineffective at informing and/or persuading (see Bullough 2017; Van Loock, Vermeir, and Geuens 2010). A study examining traffic signs in mainland China, for example, concluded that simple signs tend to be more effective ( $\mathrm{Ng}$ and Chan 2007). As another example, signage audits of libraries have shown that ineffective signs contain distracting visual clutter and “noise" (Stempler and Polger 2013; Yeaman 1989).

If signage appears cluttered, consumers may find it more difficult to understand, especially if viewing time is limited. Processing fluency (see Higgins 2000; Reber, Schwarz, and Winkielman 2004; Reber, Wurtz and Zimmermann 2004) has been defined as the ease with which new information can be processed (Schwarz 2004). It reflects the perceived ease of mental operations required to assign meaning to a stimulus and has been conceptualized as a continuum ranging from highly effortful (disfluent) to effortless (fluent) (Alter and Oppenheimer 2009). In general, increased processing fluency has positive effects on consumer evaluations by bolstering feelings of familiarity and liking (Whittlesea 1993; Whittlesea, Jacoby, and Girard 1990; King and Janiszewski 2011). In the context of signs, disfluency may cast a "dark cloud" over information and demotivate consumers to such a degree that they simply ignore the sign altogether
(Kellaris and Machleit 2016). To the extent that signage with low source attribution is simple, it may be expected to produce greater processing fluency and induce higher evaluations. Conversely, if signs with high source attribution are more complex, they may produce disfluency and lower evaluations.

Collectively, prior research on visual complexity and processing fluency suggests that consumers may value low source attribution (greater simplicity) over high source attribution (greater complexity) when processing accolade claims in signage. Of course, an alternative prediction is that level of source attribution will have negligible impact on consumer evaluations. This null effect prediction is consistent with recent work showing that people have difficulty differentiating "fake news" from real news and do not adequately discriminate between different sources, including native advertising and other content marketing, when making judgments about message credibility (see Wineburg et al. 2016; Wojdynski and Evans 2016). In contrast to both of these predictions, I make a case for a competing prediction-that consumers will prefer high source attribution in signs.

\section{THE CASE FOR HIGH SOURCE ATTRIBUTION}

Although greater effort is required to process a claim with high source attribution, as compared to one with low attribution, I contend that this difference is typically modest and so the previously conjectured benefits of low attribution in signage are limited. My proposition is that even accolade claims with high source attribution can be processed relatively quickly-with attribution serving as a salient indicator of credibility. According to most dual-process theories, consumers may either process information heuristically or systematically depending on the decision context and their own dispositional tendencies (see Chaiken et al. 1994). Heuristic processing tends to follow the peripheral route to persuasion, which leads consumers to emphasize message cues (e.g., message style, message sources) over the substantive content of a message when making evaluations (Petty and Cacioppo 1986). Certain decision-making contexts are more likely to prompt heuristic processing rather than systematic processing. For example, when processing messages quickly or when overwhelmed by information 
overload, consumers may be especially motivated to reduce search costs by expending less effort and fewer resources (Chaiken 1980; Kirmani and Rao 2000; Zhang et al. 2014). When consumers encounter outdoor or on-premise signage, it seems likely that they will attend more closely to message cues than to detailed message content. Indeed, Burke (2009) suggests that audiences are unwilling to put much cognitive effort into processing messages on a sign. I posit that level of source attribution serves as a message cue that consumers employ-even when processing heuristically-to determine the credibility of an accolade claim.

Based on this argument, higher source attribution adds credibility to an accolade, particularly when the source is itself established and reputable. Accolade claims are typically experience claims that consumers cannot easily verify without incurring a cost. As such, the incremental credibility benefit provided by source attribution may exceed the modest increase in information processing costs. Jain and Posavac (2001) have pointed out that the endorsement of an experience claim by a credible source increases "evidence sufficiency," which in turn increases the believability and persuasiveness of a message (170).

Research on accolade claims has provided corroborative evidence that consumers are able to quickly evaluate a marketing claim and make relatively sophisticated inferences. For example, consumers evaluated a bank more favorably when it claimed to be "one of the best banks" or "one of the 50 best banks," as compared to "one of the 47 best banks" or "the $47^{\text {th }}$ best bank" because they made different inferences about the bank's position on the thirdparty list in each of these conditions (Isaac, Brough, and Grayson 2016). However, another study from the same paper found that consumers under time pressure were less discriminating about different rank claims. Thus, although I hypothesize that higher source attribution will have a positive effect on consumer evaluations by increasing perceptions of credibility, this is an empirical question that has not previously been examined in the context of signage. If my hypothesis is supported, the present work would constitute the first empirical evidence that signs with accolade claims are more persuasive when they employ high source attribution.

Over three studies, I examine the effects of source attribution in accolade claims on consumer evaluations and behavioral intentions. Full stimuli for these studies are provided in the Appendix. An important assumption of this research is that higher source attribution is associated with both lower visual simplicity and greater source credibility. To verify that this was indeed the case for the stimuli used in my studies, I conducted a pretest in which 280 participants $(43.2 \%$ female, mean age $=38.54$ years, $S D=12.68)$ from an online panel (Amazon Mechanical Turk) rated either the complexity or the credibility of the sign stimuli. As shown in Table 1, both complexity ratings $(1=$ fewer words $/$ less complex, $10=$ more words $/$ more complex $)$ and source credibility ratings ( $1=$ not very believable / untrustworthy, $10=$ 
Table 1 / Pretest of Source Attribution Stimuli Across Studies

\begin{tabular}{|c|c|c|c|c|c|c|}
\hline \multirow[t]{2}{*}{$\begin{array}{l}\text { Source } \\
\text { Attribution } \\
\text { Condition } \\
\end{array}$} & \multicolumn{2}{|c|}{$\begin{array}{c}\text { Study 1: } \\
\text { Mean Rating (SD) }\end{array}$} & \multicolumn{2}{|c|}{$\begin{array}{c}\text { Study 2: } \\
\text { Mean Rating (SD) }\end{array}$} & \multicolumn{2}{|c|}{$\begin{array}{c}\text { Study 3: } \\
\text { Mean Rating (SD) }\end{array}$} \\
\hline & Complexity & Credibility & Complexity & Credibility & Complexity & Credibility \\
\hline High & $5.89(2.38)$ & $7.54(1.98)$ & $5.89(2.38)$ & $7.54(1.98)$ & $6.64(1.89)$ & $6.83(2.43)$ \\
\hline Medium & - & - & $6.00(2.45)$ & $7.39(2.02)$ & - & - \\
\hline Low & $5.43(2.50)$ & $7.32(2.09)$ & $5.14(2.68)$ & $7.28(2.05)$ & $6.18(2.45)$ & $6.40(2.70)$ \\
\hline
\end{tabular}

very believable / trustworthy) were higher in the high source attribution conditions. If source attribution simultaneously increases visual complexity and source credibility, this creates a tradeoff given that the two concepts typically have opposing effects on consumer judgments. According to my theorizing, credibility will exert greater influence in a signage context, leading higher (vs. lower) source attribution to have a more positive effect on consumer evaluations and behavioral intentions.

\section{STUDY 1}

Study 1 tests whether consumers will be more inclined to visit an award-winning restaurant when an onpremise sign is high versus low in source attribution. I predict that when source attribution of an accolade is high, behavioral intentions to visit the restaurant will be higher.

\section{Method}

A total of 221 participants from a large American university $(23.1 \%$ female, mean age $=20.58$ years, $S D=1.00)$ completed this study in exchange for partial course credit. This study involved a singlefactor between-participants design with two source attribution conditions (high vs. low).

Participants learned of a restaurant nearby that they had never visited. They were shown signage displayed near the entrance of the restaurant that they had noticed one day while walking home. Participants in both conditions saw a storefront's brick façade on which two signs were affixed. The sign on the right, which was identical for all participants, displayed the name of the restaurant, "The Greathouse of Pizza," and the restaurant's logo. The sign on the left appeared to be a plaque depicting the restaurant's selection as a "Reader's Choice 2019" award-winner and its selection on a "10 Best" list. My source attribution manipulation involved the inclusion or exclusion of a single word on this plaque. Specifically, the plaque shown to participants in the high attribution condition included the words USA Today, presumably indicating that the accolade was bestowed by the national newspaper. For participants in the low attribution condition, "USA" remained on the sign but the word "Today" was removed. This represents a conservative test of my prediction that consumers attend closely to source attribution in on-premise signage, as the only difference between conditions was a single word.

After qualitatively describing their reaction to the signage in a text box, participants responded to the key dependent variable. Specifically, participants indicated the extent to which this signage affected their likelihood to visit the restaurant $(1=$ less likely to visit, $10=$ more likely to visit). Following this behavioral intention question, participants evaluated the credibility of the restaurant by responding to three items ( 1 = not very honest / not very trustworthy / not very believable, $10=$ very honest / very trustworthy / very believable), which were combined to form a composite perceived credibility measure $(\alpha=.87)$.

Perceived restaurant credibility was included as a potential mediator for the relationship between source attribution condition and restaurant visit likelihood. From a construct validity perspective, it is worth noting that although source credibility is inherently related to level of source attribution, restaurant credibility is a distinct and orthogonal construct and therefore a valid potential mediator. I predict that because it is endorsed by a credible source, an organization will itself be viewed as more credible. This prediction is consistent with prior work documenting positive spillover effects of reputation (see Kilduff and Krackhardt 1994). 
Table 2 / Dependent Variables Across Studies

\begin{tabular}{cccc}
\hline $\begin{array}{c}\text { Source Attribution } \\
\text { Condition }\end{array}$ & $\begin{array}{c}\text { Study 1 (Student Sample): Mean } \\
\text { Restaurant Visit Likelihood } \\
(\text { SD) }\end{array}$ & $\begin{array}{c}\text { Study 2 (MTurk Sample): } \\
\text { Mean Restaurant Evaluation } \\
\text { (SD) }\end{array}$ & $\begin{array}{c}\text { Study 3 (MTurk Sample): } \\
\text { Mean Law Firm Evaluation } \\
\text { (SD) }\end{array}$ \\
\hline High & $8.18(1.93)$ & $8.10(1.81)$ & $5.44(2.57)$ \\
Medium & - & $7.53(1.97)$ & - \\
Low & $7.58(2.17)$ & $7.11(2.12)$ & $4.80(2.74)$ \\
\hline
\end{tabular}

\section{Results}

I expected that greater source attribution would make participants more likely to visit the restaurant. In line with this prediction, likelihood to visit the restaurant was higher among participants in the high source attribution condition $(M=8.18, S D=1.93, N=$ $110)$ versus the low source attribution condition $(M$ $=7.58, S D=2.17, N=111 ; t(219)=2.19, p=.029)$. Means for the key dependent variable in each studybehavioral intentions and/or consumer evaluationsare displayed in Table 2 (above).

In addition to being more likely to visit the restaurant, participants in the high source attribution condition $(M=7.54, S D=1.67, N=110)$ rated the restaurant as more credible than participants in the low source credibility condition $(M=7.04, S D=1.72, N=111$; $t(219)=2.21, p=.028)$.

Finally, I conducted a mediation analysis using the PROCESS macro (Model 4) to test whether perceived credibility of the restaurant mediated the effect of source attribution on likelihood to visit the restaurant (Hayes 2017). This mediation analysis utilized bootstrapping with repeated extraction of 10,000 samples. For this purpose, the high attribution condition was coded as ' 1 ,' and the low attribution condition was coded as '0,' with likelihood to visit the restaurant as the dependent variable. I included perceived credibility as a potential mediator in the model. Results of the mediation analysis indicated that the indirect effect of source attribution through perceived credibility was positive $(B=.41, S E=.19)$ and statistically different from zero (95\% CI: .04, .80). Taken together, these results indicate that source attribution impacted perceived credibility of the restaurant, which influenced likelihood to visit.

\section{Discussion}

The results of Study 1 provide initial evidence that consumers attend to source information in onpremise signs. As a result, high source attribution is beneficial when an organization wishes to attract potential customers because it bolsters the organization's own credibility.

\section{STUDY 2}

In Study 2, I attempt to replicate the findings of Study 1 using a different sample and a different operationalization of source attribution, this time with three levels. In the present research, I adopt a broad definition of source attribution that not only includes how prominently (if at all) the thirdparty entity that bestowed the accolade upon the organization is mentioned, but also the amount of detail given about the accolade itself. In Study 2, the name of the third-party entity is provided in both the high and medium source attribution conditions, but the medium attribution claim provides fewer details about the accolade. Finding that consumer evaluations of the medium attribution claim fall in between evaluations of the other two claims would indicate that consumers are sensitive to both the identity of the source and the amount of information communicated about the accolade, which I consider two facets of source attribution.

\section{Method}

A total of 255 participants from an online panel (Amazon Mechanical Turk) (55.5\% female, mean age $=39.59$ years, $S D=14.18$ ) completed this study in exchange for payment. This study involved a singlefactor between-participants design with three source attribution conditions (high vs. medium vs. low).

Study 2 used the same restaurant context- "The Greathouse of Pizza" - as Study 1 and the procedure 
was identical, except for the manipulation involving the attribution of the award. The high attribution condition in Study 2 resembled the corresponding condition in Study 1, in that it mentioned that the USA Today had included the restaurant in its " 10 Best" list. Those in the medium attribution condition also learned that the award came from the USA Today, but no mention of the "10 Best" list was provided. Participants in the low attribution condition learned that the award involved selection in a "10 Best" list, but the source was missing, in that the name of the newspaper was not mentioned at all.

After qualitatively describing their reaction to the signage in a text box, participants responded to the key dependent variables. Specifically, participants evaluated the restaurant by responding to three items $(1=$ less likely to visit / more negative evaluation / less favorable attitude, $10=$ more likely to visit / more positive evaluation / more favorable attitude), the first of which is a behavioral intention indicator. These three items were combined to form a composite restaurant evaluation measure $(\alpha=.95)$. Subsequently, participants provided their opinion of the Reader's Choice award that the restaurant had received by responding to three items $(1=$ not very impressive / not a major achievement / not a great honor, 10 = very impressive / a major achievement/a great honor). These three items were combined to form a composite award prestige measure $(\alpha=$ .97). Finally, participants evaluated the credibility of the restaurant by responding to three items $(1=$ not very honest / not very trustworthy / not very believable, 10 = very honest / very trustworthy / very believable), which were combined to form a composite perceived credibility measure $(\alpha=.96)$.

\section{Results}

I predicted that greater source attribution would lead to higher evaluations of the restaurant, which would be driven by higher award prestige and higher credibility of the restaurant. A one-way ANOVA confirmed that participants' restaurant evaluations differed significantly by condition $(F(2,252)=5.39$, $\left.p=.005 ; \eta_{p}^{2}=.041\right)$. Restaurant evaluations were higher among participants in the high attribution condition $(M=8.10, S D=1.81, N=85)$ as compared to either the medium $(M=7.53, S D=1.97, N=86$; $t(252)=1.90, p=.058)$ or low attribution condition $(M=7.11, S D=2.12, N=84 ; t(252)=3.27, p=.001)$, although the former contrast attained only marginal significance. Although evaluations were directionally higher among participants in the medium attribution condition versus the low attribution condition (see Table 2), this contrast was non-significant $(t(252)=$ $1.38, p=.17$ ). Thus, my subsequent analysis focuses primarily on the high evaluation condition versus the other two conditions.

I conducted two additional one-way ANOVAs, first with award prestige as the dependent variable and then with perceived credibility as the dependent variable. Both ANOVAs returned a similar result. Specifically, the analysis confirmed that participants' award prestige ratings differed marginally by condition $(F(2$, $\left.252)=2.62, p=.075 ; \eta_{p}^{2}=.020\right)$. Means were $7.62(S D$ $=2.28), 7.12(S D=2.41)$, and $6.77(S D=2.55)$ in the high, medium, and low source attribution conditions, respectively. Likewise, participants' perceptions of the restaurant's credibility differed significantly by condition $\left(F(2,252)=3.84, p=.023 ; \eta_{p}^{2}=.030\right)$. Means were $7.85(S D=1.99), 7.40(S D=2.07)$, and $6.97(S D=2.12)$ in the high, medium, and low source attribution conditions, respectively.

Finally, I conducted a mediation analysis using the PROCESS macro (Model 6) to test whether award prestige and/or perceived credibility mediated the effect of source attribution on restaurant evaluations (Hayes 2017). This mediation analysis utilized bootstrapping with repeated extraction of 10,000 samples. For this purpose, all the conditions were grouped into two source attribution cells (high attribution coded as ' 1 ,' medium and low attribution coded as ' 0 '), with the composite restaurant evaluation measure as the dependent variable. Award prestige and perceived credibility were included as potential mediators in the model. Results of the mediation analysis indicated that the indirect effect of source attribution through perceived credibility was positive $(B=.37, S E=.17)$ and statistically different from zero (95\% CI: .06, .73). The indirect effect of condition through award prestige, however, 
was not statistically different from zero $(B=.01, S E=.04 ; 95 \%$ CI: $-.07, .10)$. Furthermore, I found evidence of serial mediation from source attribution to perceived credibility, perceived credibility to award prestige, and from award prestige to restaurant evaluation $(B=.14, S E=.08$; 95\% CI: .01, .31). Taken together, these results indicate that attribution impacted perceived credibility of the restaurant, which influenced award prestige, which in turn affected restaurant evaluations.

\section{Discussion}

The results of Study 2 provide additional evidence that high source attribution in on-premise signs is beneficial when firms wish to communicate an accolade to potential customers. Specifically, Study 2 shows that high source attribution increases perceived credibility of the firm, which makes the accolade appear even more impressive. Furthermore, I find that consumers are sensitive to two facets of source attribution, namely the identity of the source and the amount of information communicated about the accolade.

\section{STUDY 3}

Although the results of Studies 1 and 2 are consistent with my theorizing, participants were not under any time pressure when viewing the signs in either study. Given the fact that most consumers typically view certain types of signs, such as billboards, for only a few seconds, Study 3 tests whether the value of high source attribution will be observed even when participants are given only a few seconds to process the sign's content. The results of prior research suggest that consumers are able to quickly make sophisticated inferences from marketing communications and so high source attribution claims may still outperform low source attribution claims even under time pressure; however, this evidence is somewhat inconsistent and so my a priori hypothesis is somewhat tentative (see Isaac, Brough, and Grayson 2016).

\section{Method}

A total of 506 participants from an online panel (Amazon Mechanical Turk) $(54.2 \%$ female, mean age $=36.64$ years, $S D=13.02)$ completed this study in exchange for payment. This study involved a single-factor betweenparticipants design with two source attribution conditions (high vs. low).

To ensure that participants felt sufficiently involved in the scenario, they learned that they had recently been in a traffic accident with another car in which they had sustained an injury. They disagreed with the other driver as to who was at fault and were considering hiring a lawyer to settle the dispute.

Next, they were shown an outdoor sign that theyhad purportedly encountered while driving to work. All participants encountered a billboard that stated the name of the law firm, Domengeaux Wright, Roy, and Edwards, included contact information, and touted the accolade "Best Lawyers, Best Law Firms." The billboard shown to participants in the high attribution condition included the words U.S. News and World Report 2018, presumably indicating 
that this honor came from the national magazine. The billboard shown to participants in the low attribution condition did not include these words. However, the font size of the accolade claim was three times the size as the claim in the high attribution condition. This manipulation was meant to more strongly reflect the inherent tradeoff between simplicity and credibility in signage. Specifically, because it did not contain source attribution information, the low attribution sign could utilize this extra space to increase the visibility of the accolade claim itself.

To simulate the experience of driving past a billboard, the law firm billboard was shown on the screen quickly (i.e., for three seconds), at which point participants automatically advanced to a new screen where they answered follow-up questions. After qualitatively describing their reaction to the billboard in a text box, participants responded to the key dependent variable. Specifically, participants evaluated the law firm by responding to three items ( $1=$ less likely to hire / more negative evaluation / less favorable attitude, 10 $=$ more likely to hire / more positive evaluation / more favorable attitude), the first of which is a behavioral intention indicator. These three items were combined to form a composite law firm evaluation measure $(\alpha=$ .97). Subsequently, participants were asked to indicate whether the billboard had mentioned an honor that the law firm had received (Yes / No). If they answered affirmatively, participants were asked to provide their opinion of the honor that the law firm had received by responding to three items $(1=$ not very impressive / not a major achievement / not a great honor, $10=$ very impressive / a major achievement / a great honor). These three items were combined to form a composite award prestige measure $(\alpha=.96)$. Finally, participants evaluated the credibility of the law firm by responding to three items $(1=$ not very honest / not very trustworthy / not very believable, $10=$ very honest / very trustworthy / very believable), which were combined to form a composite perceived credibility measure $(\alpha=.92)$.

\section{Results}

I predicted that greater source attribution would lead to higher evaluations of the law firm, which would be driven by higher credibility of the law firm. In line with this prediction, evaluations of the law firm were higher among participants in the high source attribution condition $(M=5.44, S D=2.57, N=248)$ versus the low source attribution condition $(M=4.80$, $S D=2.74, N=258 ; t(504)=2.71, p=.007)($ see Table 2$)$.

Furthermore, participants in the high source attribution condition $(M=6.15, S D=2.04, N=248)$ rated the law firm as more credible than participants in the low source credibility condition $(M=5.38, S D$ $=2.32, N=258 ; t(504)=3.97, p<.001)$.

Next, I conducted a mediation analysis using the PROCESS macro (Model 4) to test whether perceived credibility mediated the effect of source attribution on law firm evaluations (Hayes 2017). This mediation analysis utilized bootstrapping with repeated extraction of 10,000 samples. For this purpose, the high attribution condition was coded as ' 1 ', and the low attribution condition was coded as '0,' with law firm evaluation as the dependent variable. I included perceived credibility as a potential mediator in the model. Results of the mediation analysis indicated that the indirect effect of source attribution through perceived credibility was positive $(B=.75, S E=.19)$ and statistically different from zero (95\% CI: .38, 1.12). Taken together, these results indicate that source attribution impacted perceived credibility of the law firm, which influenced law firm evaluations.

I also examined whether source attribution affected participants' likelihood of recognizing that the law firm had received an honor. Of participants in the high attribution condition, 62.5\% (155 of 248) reported noticing the honor as compared to $32.6 \%$ of participants (84 of 258) in the low attribution condition, a statistically significant difference $\left(\chi^{2}(1)=\right.$ $45.49, p<.001)$. Among participants who recognized that the law firm had received an honor, ratings of award prestige did not differ $\left(M_{\text {high_attribution }}=6.44\right.$, $S D=2.23, N=155$ vs. $M_{\text {low_attribution }}=6.56, S D=2.47$, $N=84 ; t(237)=-.37, p=.71)$. This analysis suggests that even when viewing a sign quickly, consumers are sensitive to source attribution.

\section{Discussion}

Study 3 shows that consumers attend to source 
information in outdoor signs, time notwithstanding. Furthermore, my analysis suggests that without sufficient attribution, an accolade claim may not even be encoded as a legitimate honor from a thirdparty entity and may therefore be dismissed as noncredible. Finally, this study provides corroborative evidence that source information in outdoor signs boosts evaluations by increasing perceptions of an organization's own credibility, even when higher source attribution reduces visibility of the accolade.

\section{GENERAL DISCUSSION}

Across three studies, I provide converging evidence that consumers respond favorably to higher source attribution when given an accolade claim, even in the context of signage where visual simplicity and processing fluency are of paramount importance. As shown in Table 2, this effect holds for different samples and stimuli, as well as for both behavioral intentions and composite evaluation measures.

I further show that the positive effects of higher source attribution result from greater perceived credibility of the organization, which in turn increases the prestige of the award. It is worth noting that this credibility measure relates to the organization, not the source. While it may be expected that increased source attribution would generally lead to increased source credibility (as confirmed by my pretest), my finding is that an organization that receives an award and displays a sign with high source attribution is also viewed as more credible. As demonstrated in Study 3 , insufficient source attribution may lead consumers to not even recognize an accolade claim as a "true" award coming from an impartial third-party. Of course, the positive effects of source attribution are likely to be contingent on the third-party entity itself being familiar and credible to consumers and might not hold if the third-party is unknown or considered non-credible. Indeed, Isaac and Grayson (2020) recently showed that consumer ratings differed when an accolade was attributed to a reputable news source (i.e., BBC) as compared to a disreputable tabloid (i.e., TMZ). It is reasonable to assume that high source attribution will only boost evaluations when the source is itself considered credible.
Future research might identify additional boundary conditions for the effects observed in this article. For example, it seems likely that signs with an abundance of detailed and extraneous information about an award or the source of the award would be penalized rather than rewarded by consumers. Additionally, participants in my three studies either had unlimited time to review an accolade claim (Studies 1 and 2) or assumed a relatively high level of involvement in the given scenario (Study 3). It is possible that under different conditions, signs with low source attribution might outperform (or at least perform equally well as) signs with high source attribution. Relatedly, it is possible that consumers have different preferences for level of source attribution when viewing on-premise as compared to outdoor signs because they are at different stages of the consumer decision journey. In practice, on-premise sign viewers are more likely to be actual customers of the organization, given that they are already on site, whereas viewers of outdoor signs may only be prospective customers. As such, on-premise sign viewers may naturally be more involved with the organization and therefore especially amenable to high source attribution. Given the hypothetical nature of the studies reported in this article, I was unable to distinguish between consumer responses to source attribution in outdoor signs versus on-premise signs. I encourage future researchers to employ field experiments to provide more nuanced insights into potential distinctions based on sign type.

Although I found that low source attribution in signage tends to produce lower evaluations, it is important to note that not all low attribution signs are the same. For instance, some of the examples in Figure 2 of accolade claims with low source attribution might be interpreted as "tongue-in-cheek" or humorous puffery claims, as opposed to unsupported and noncredible accolades (Cowley 2006). Whereas the latter class of low attribution claims seem to adversely affect consumer judgments, it may be that consumers appreciate the attempt at humor in a puffery claim and actually reward organizations for this type of low attribution claim. Future research might investigate whether different types of low attribution claims have different effects on consumer evaluations. 
Finally, prior research has demonstrated the role of norms in communication and has shown that minor variations in a communicator's word choice or framing can affect consumer evaluations and decisions (Grice 1975; McKenzie and Nelson 2003; Sher and McKenzie 2006). For certain types of signs, it may be more or less normative to provide source attribution information. Furthermore, certain ways of conveying source attribution-using a text description versus a logo of the third-party entity, for example-may be more or less effective. I encourage persuasion researchers to explore these intriguing avenues for future research.

\section{REFERENCES}

Alter, A. L. and Oppenheimer, D. M. (2009). Uniting the tribes of fluency to form a metacognitive nation. Personality and Social Psychology Review 13(3), 219-235.

Anderson, R. E. and Jolson, M. A. (1980). Technical wording in advertising: implications for market segmentation. Journal of Marketing 44, 57-66.

Balasubramanian, S. K., Mathur, I., and Thakur, R. (2005). The impact of high-quality firm achievements on shareholder value: Focus on Malcolm Baldrige and JD Power and Associates awards. Journal of the Academy of Marketing Science 33(4), 413-422.

Bullough, J. (2017). Factors affecting sign visibility, conspicuity, and legibility: Review and annotated bibliography. Interdisciplinary Journal of Signage and Wayfinding 1(2), 2-25.

Bullough, J. D. (2019). Brief Communication: Impact of Sign Panel Luminance on Visual Comfort. Interdisciplinary Journal of Signage and Wayfinding 3(2), 3-7.

Burke, R. R. (2009). Behavioral effects of digital signage. Journal of Advertising Research 49(2), 180-185.

Capital Outdoor. (2020). "Measuring Billboard Effectiveness." Capitol Outdoor. https://www.capitoloutdoor.com/measuring-billboardadvertising-effectiveness/

Chaiken, S. (1980). Heuristic versus systematic information processing and the use of source versus message cues in persuasion. Journal of Personality and Social Psychology 39(5), 752-766.

Chaiken, S. and Maheswaran, D. (1994). Heuristic processing can bias systematic processing: effects of source credibility, argument ambiguity, and task importance on attitude judgment. Journal of Personality and Social Psychology, 66(3), 460-473.

Chamblee, R., Gilmore, R., Thomas, G., Soldow, G. (1993). When copy complexity can help ad readership. Journal of Advertising Research 33(3), 23-28.

Clear Channel Outdoor. (2019). “Guidelines \& specifications for designing out of home." Clear Channel Outdoor. https:// clearchanneloutdoor.com/get-creative/

Cowley, E. (2006). Processing exaggerated advertising claims. Journal of Business Research 59(6), 728-734.

Dean, D. H. and Biswas, A. (2001). Third-party organization endorsement of products: An advertising cue affecting consumer prepurchase evaluation of goods and services. Journal of Advertising 30(4), 41-57.

Garvey, P. M., Zineddin, A. Z., Porter, R. J., and Pietrucha, M. T. (2018). Real world on-premise sign visibility: The impact of the driving task on sign detection and legibility. Washington DC: United States Signage Council Foundation and The US Department of Transportation.

Grice, H. P. (1975). Logic and conversation. Speech Arts. Leiden: Brill.

Hayes, A. F. (2017). Introduction to Mediation, Moderation, and Conditional Process Analysis: A Regression-Based Approach. New York: Guilford Press.

Higgins, E. T. (2000). Making a good decision: value from fit. American Psychologist 55(11), 1217-1230.

Isaac, M. S., Brough, A. R., and Grayson, K. (2016). Is top 10 better than top 9? The role of expectations in consumer response to imprecise rank claims. Journal of Marketing Research 53(3), 338-353.

Isaac, M. S. and Grayson, K. (2020). Priming skepticism: Unintended consequences of one-sided persuasion knowledge access. Psychology and Marketing 37(3), 466-478. 
Jain, S. P. and Posavac, S. S. (2001). Prepurchase attribute verifiability, source credibility, and persuasion. Journal of Consumer Psychology 11(3), 169-180.

Kellaris, J. J. and Machleit, K. A. (2016). Signage as marketing communication: A conceptual model and research propositions. Interdisciplinary Journal of Signage and Wayfinding 1(1).

Kilduff, M. and Krackhardt, D. (1994). Bringing the individual back in: A structural analysis of the internal market for reputation in organizations. Academy of Management Journal 37(1), 87-108.

King, D. and Janiszewski, C. (2011). The sources and consequences of the fluent processing of numbers. Journal of Marketing Research 48(2), 327-341.

Kirmani, A. and Rao, A. R. (2000). No pain, no gain: A critical review of the literature on signaling unobservable product quality. Journal of Marketing 64(2), 66-79.

Lowrey, T. M. (1998). The effects of syntactic complexity on advertising persuasiveness. Journal of Consumer Psychology 7(2), 187-206. MacInnis, D. J., Moorman, C., and Jaworski, B. J. (1991). Enhancing and measuring consumers' motivation, opportunity, and ability to process brand information form ads. Journal of Marketing 55(October), 32-53.

McKenzie, C. R. and Nelson, J. D. (2003). What a speaker's choice of frame reveals: Reference points, frame selection, and framing effects. Psychonomic Bulletin and Review 10(3), 596-602.

Morrison, B. J. and Dainoff, M. J. (1972). Advertisement complexity and looking time. Journal of Marketing Research 9(4), 396-400.

Ng, A. W. Y. and Chan, A. H. S. (2007). Cognitive design features on traffic signs. In: Recent Advances in Engineering and Computer Science, ed. S. Ao. Hong Kong: Newswood Limited.

Petty, R. E. and Cacioppo, J. T. (1986). The elaboration likelihood model of persuasion. In: Communication and Persuasion, Springer Series in Social Psychology. New York: Springer.

Phillips, B. J. (1997). Thinking into it: consumer interpretation of complex advertising images. Journal of Advertising 26 (2), 77-87.

Reber, R., Schwarz, N., and Winkielman, P. (2004). Processing fluency and aesthetic pleasure: Is beauty in the perceiver's processing experience? Personality and Social Psychology Review 8(4), 364-382.

Reber, R., Wurtz, P., and Zimmermann, T. D. (2004). Exploring "fringe" consciousness: The subjective experience of perceptual fluency and its objective bases. Consciousness and Cognition 13(1), 47-60.

Rossiter, J. R. and Percy, L. (1983). Visual communication in advertising, In: Information Processing Research in Advertising, ed. R. Harris. Hillsdale: Lawrence Erlbaum Associates.

Schwarz, N. (2004). Metacognitive experiences in consumer judgment and decision making. Journal of Consumer Psychology 14(4), 332-348.

Shah, K. (2020). "Food \& Wine best new restaurants 2020." Food and Wine, 16 June. https://www.foodandwine.com/travel/restaurants/ best-new-restaurants

Sher, S. and McKenzie, C. R. (2006). Information leakage from logically equivalent frames. Cognition 101(3), 467-494.

Shuptrine, F. K. and McVicker, D. D. (1981). Readability levels of magazine ads. Journal of Advertising Research 21(5), 45-51.

Stempler, A. F. and Polger, M. A. (2013). Do you see the signs? Evaluating language, branding, and design in a library signage audit. Public Services Quarterly 9(2), 121-135.

Van Loock, N., Vermeir, I., and Geuens, M. (2010). Advertising repetition and complexity of digital signage advertisements: simplicity rules! In: 39th European Marketing Academy Conference. Ghent: Department of Marketing.

Whittlesea, B. W. (1993). Illusions of familiarity. Journal of Experimental Psychology: Learning, Memory, and Cognition 19(6), 1235-1253. Whittlesea, B. W., Jacoby, L. L., and Girard, K. (1990). Illusions of immediate memory: Evidence of an attributional basis for feelings of familiarity and perceptual quality. Journal of Memory and Language 29(6), 716-732.

Wineburg, S., McGrew, S., Breakstone, J., and Ortega, T. (2016). Evaluating information: The cornerstone of civic online reasoning. Stanford Digital Repository. https://purl.stanford.edu/fv751yt5934

Wojdynski, B. W. and Evans, N. J. (2016). Going native: Effects of disclosure position and language on the recognition and evaluation of online native advertising. Journal of Advertising, 45(2), 157-168.

Yeaman, A. R. (1989). Vital signs: Cures for confusion. School Library Journal 35(15), 23-27.

Zhang, K. Z., Zhao, S. J., Cheung, C. M., and Lee, M. K. (2014). Examining the influence of online reviews on consumers' decisionmaking: A heuristic-systematic model. Decision Support Systems 67, 78-89. 


\section{APPENDIX}

\section{Study 1}

Suppose there is a pizza place nearby that you have never visited before. One day, as you are walking home, you notice the following signage near the entrance of the restaurant.

\section{High Source Attribution}

Next to a sign with the name and logo of the pizza place, "The Greathouse of Pizza, is another sign which looks like a plaque. This sign indicates that the pizza place won a Reader's Choice "10 Best" award in 2019. The sign indicates that this honor was bestowed upon The Greathouse of Pizza by the USA Today.

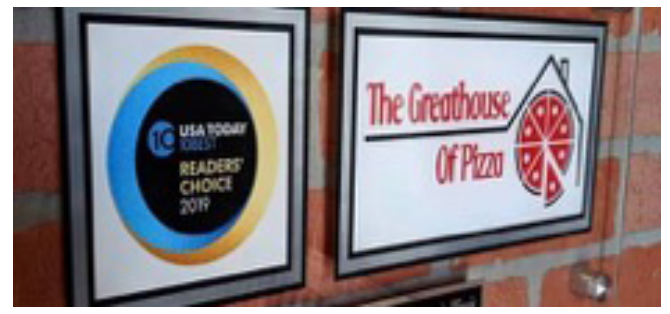

\section{Low Source Attribution}

Suppose there is a pizza place nearby that you have never visited before. One day, as you are walking home, you notice the signage near the entrance of the restaurant.

Next to a sign with the name and logo of the pizza place, "The Greathouse of Pizza," is another sign which looks like a plaque. This sign indicates that the pizza place won a Reader's Choice award in 2019.

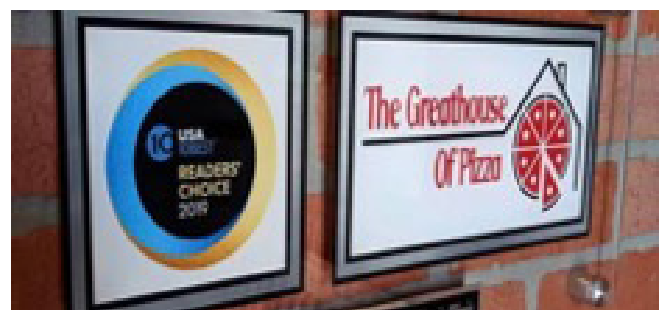

\section{Measures}

What is your reaction to the Reader's Choice signage (the sign on the left)? [Open-Ended Text Box]

\section{BEHAVIORAL INTENTION}

How does this signage affect your opinion of the pizza place? $1=$ less likely to visit, $10=$ more likely to visit

\section{PERCEIVED CREDIBILITY}

Based on its signage, what do you think of the pizza place?

$1=$ not very honest, $10=$ very honest

$1=$ not very trustworthy, 10 = very trustworthy

$1=$ not very believable, $10=$ very believable

\section{Study 2}

Suppose there is a pizza place nearby that you have never visited before. One day, as you are walking home, you notice the following signage near the entrance of the restaurant.

\section{High Source Attribution}

Next to a sign with the name and logo of the pizza place, "The Greathouse of Pizza, is another sign which looks like a plaque. This sign indicates that the pizza place won a Reader's Choice "10 Best” award in 2019. The sign indicates that this honor was bestowed upon The Greathouse of Pizza by the USA Today, a popular national newspaper.

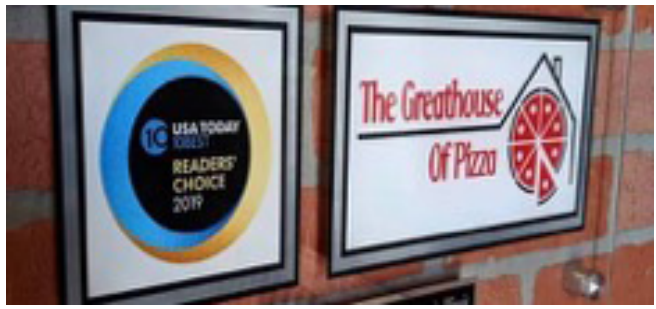

\section{Medium Source Attribution}

Next to a sign with the name and logo of the pizza place, "The Greathouse of Pizza," is another sign which looks like a plaque. This sign indicates that the pizza place won a Reader's Choice award in 2019. The sign indicates that this honor was bestowed upon The Greathouse of Pizza by the USA Today, a popular national newspaper.

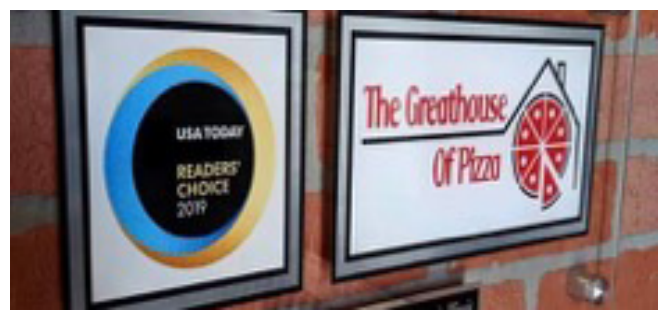




\section{Low Source Attribution}

Next to a sign with the name and logo of the pizza place, "The Greathouse of Pizza," is another sign which looks like a plaque. This sign indicates that the pizza place won a Reader's Choice "10 Best" award in 2019. The sign does not indicate who bestowed this honor upon The Greathouse of Pizza.

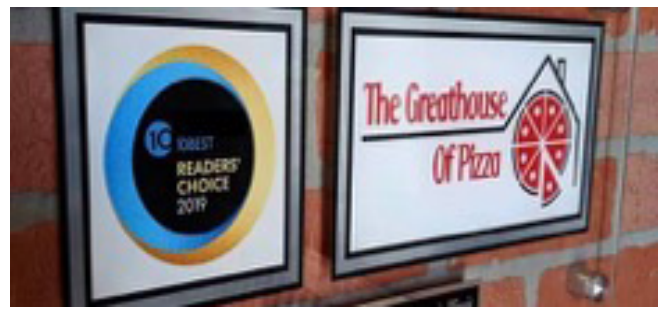

\section{Measures}

What is your reaction to the Reader's Choice signage (the sign on the left)? [Open-Ended Text Box]

\section{RESTAURANT EVALUATION}

How does this signage affect your opinion of the pizza place?

$1=$ less likely to visit, $10=$ more likely to visit

$1=$ more negative evaluation, $10=$ more positive evaluation

1 = less favorable attitude, $10=$ more favorable attitude

\section{AWARD PRESTIGE}

What is your opinion of the pizza place's Reader's Choice award?

$1=$ not very impressive, $10=$ very impressive

$1=$ not a major achievement, $10=$ a major achievement

$1=$ not a great honor, $10=$ a great honor

PERCEIVED CREDIBILITY

Based on its signage, what do you think of the pizza place?

$1=$ not very honest, $10=$ very honest

$1=$ not very trustworthy, $10=$ very trustworthy

$1=$ not very believable, $10=$ very believable

\section{Study 3}

Suppose you were recently in a traffic accident in which you sustained an injury. You and the other driver disagree as to whose fault the accident was. You are considering hiring a lawyer.

As you are driving to work, you pass a billboard. Press -> to see the billboard. It will be shown on the screen quickly (for 3 seconds) and then you will advance to a new screen where you will answer a few questions.

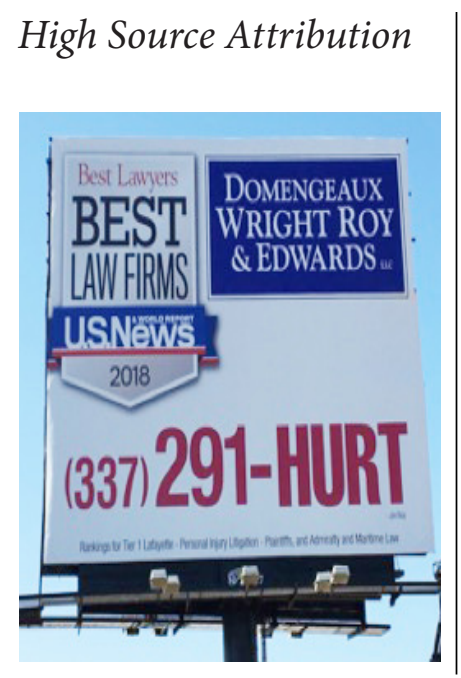

Low Source Attribution

\section{Measures}

What was your reaction to the billboard? [Open-Ended Text Box]

\section{LAW FIRM EVALUATION}

How does the billboard affect your opinion of the law firm

(Domengeaux Wright Roy \& Edwards)?

$1=$ less likely to hire, $10=$ more likely to hire

$1=$ more negative evaluation, $10=$ more positive evaluation

$1=$ less favorable attitude, $10=$ more favorable attitude

Did the billboard mention an honor that the law firm

(Domengeaux Wright Roy \& Edwards) had received?

$1=$ Yes, $2=$ No

\section{[IF YES]}

What do you think of the honor that the law firm (Domengeaux

Wright Roy \& Edwards) received?

$1=$ not very impressive, $10=$ very impressive

$1=$ not a major achievement, $10=$ a major achievement

$1=$ not a great honor, $10=$ a great honor

Did the billboard mention the third-party organization or magazine that bestowed the honor on the law firm (Domengeaux Wright Roy \& Edwards)?

\section{PERCEIVED CREDIBILITY}

Based on its billboard, what do you think of the law firm

(Domengeaux Wright Roy \& Edwards)?

$1=$ not very honest, $10=$ very honest

$1=$ not very trustworthy, $10=$ very trustworthy

$1=$ not very believable, $10=$ very believable 